\title{
Spektrum der Phlebologie
}

\section{Liebe Leserinnen und Leser,}

wie im Hefttitel schon angedeutet, bildet die aktuelle Ausgabe das vielseitige Spektrum der Phlebologie zu aktuellen und praxisrelevanten Fragestellungen ab: Im ersten Beitrag weist T. Hirsch, Halle (Saale), auf wissenswerte Effekte einer Kompressionstherapie bei Patienten mit Herzinsuffizienz hin: Denn bei strenger Indikationsstellung stellt eine Herzinsuffizienz je nach NYHAStadium nicht schon per se eine absolute Kontraindikation für Kompressionstherapie oder manuelle Lymphdrainage dar.

T. Bertsch und G. Erbacher, Hinterzarten, fokussieren in diesem zweiten Beitrag über die „Mythen des Lipödems“ (Teil 1 siehe Ausgabe 2-2018) auf das „Ödem im Lipödem". Hier wird die manuelle Lymphdrainage als essenzielle und regelmäßig durchzuführende Standardtherapie von den Autoren kritisch hinterfragt.

Es folgen zwei Kasuistiken: P.-M. Baier und Z. T. Misczcak, Bad Neuenahr, stellen zwei Patienten vor, die initial wegen einer Rezidivvarikose operativ behandelt wurden und postoperativ therapieresistente Lymphozelen entwickelt haben. Über einen fulminanten Verlauf des Trousseau-Syndroms berichten T. Silber, K. Schweinzer und A. Strölin, Tübingen.

Die zunehmende Verwendung von direkten oralen Antikoagulanzien (DOAKs bzw. NOAKs) erfordert spezielle Kenntnisse im Umgang mit schwierigen klinischen
Situationen beim Patienten. Daher befassen sich in dieser Ausgabe gleich zwei Übersichtsarbeiten mit diesem wichtigen Themenkomplex: J. Koscielny, C. Rosenthal und C. von Heymann, Berlin, beschreiben den Einsatz von DOAKs in der elektiven Chirurgie. In der zweiten Übersichtsarbeit berichten S. Kücükköylü und L. C. Rump, Düsseldorf, über den Einsatz von DOAKs bei Patienten mit chronischen Beeinträchtigungen der Nierenfunktion.

In der Rubrik Junge Phlebologie: Fortbildung in Bildern erklären A. L. Recke und B. Kahle, Lübeck, die "Sklerosierungstherapie von Besenreisern" für Einsteiger: In anschaulichen Abbildungen wird das leitliniengerechte Vorgehen bei kleinkalibrigen Varizen dargestellt.

Zuletzt noch zwei Sätze in eigener Sache: Im Dezember 2017 habe ich die redaktionelle Betreuung der Phlebologie übernommen. Ich freue mich schon sehr über einen regen Austausch mit Ihnen und möchte Sie an dieser Stelle dazu ermuntern, sich bei Fragen, Wünschen oder Anregungen jederzeit bei mir zu melden (E-Mail: jan.hueber@schattauer.de).

Viel Freude beim Lesen dieser Ausgabe der Phlebologie!

Ihr

Dr. med. Jan Hueber

Redaktion Phlebologie

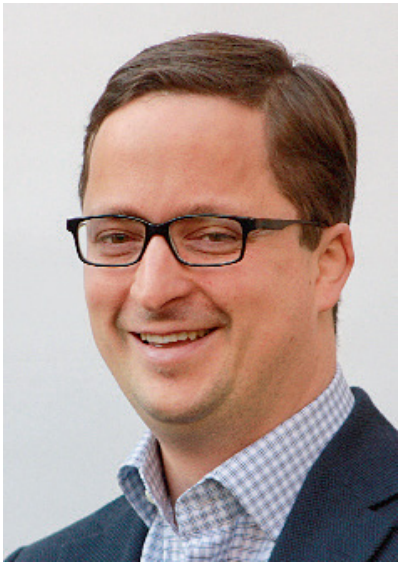

Dr. Jan Hueber, Stuttgart 